\section{Three-Dimensional Crystallographic Analysis Beyond EBSD Mapping: The Next Dimension}

\author{
J.J.L. Mulders*, and A. Gholinia**
}

*FEI Electron Optics, Eindhoven, The Netherlands, jjm@nl.feico.com

**HKL Technology, Hobro, Denmark, gholinia@hkltechnology.com

\section{Introduction}

Since the early 1990's EBSD (Electron Back Scatter Diffraction) has been developed to become a mature crystallographic analysis technique at the micro and nano-scale. It is applied in a SEM (Scanning Electron Microscope) on samples with a very smooth and clean surface. It provides quantitative orientations of individual grains, and by mapping a larger area, multiple grain analysis, texture, and grain boundaries can be examined. As the useful information for the EBSD technique comes from a very shallow depth in the material (10 to $20 \mathrm{~nm}$ ), it is a surface analysis technique showing lateral 2D distributions of crystal orientations.

Ascertaining the crystal orientation distribution in the third dimension (into the surface - the $Z$-direction) can be obtained at larger scale $(\mathrm{mm})$ by 3D-XRD (X-Ray Diffraction), whereas the very small scale $(\mathrm{nm})$ is covered by TEM (Transmission
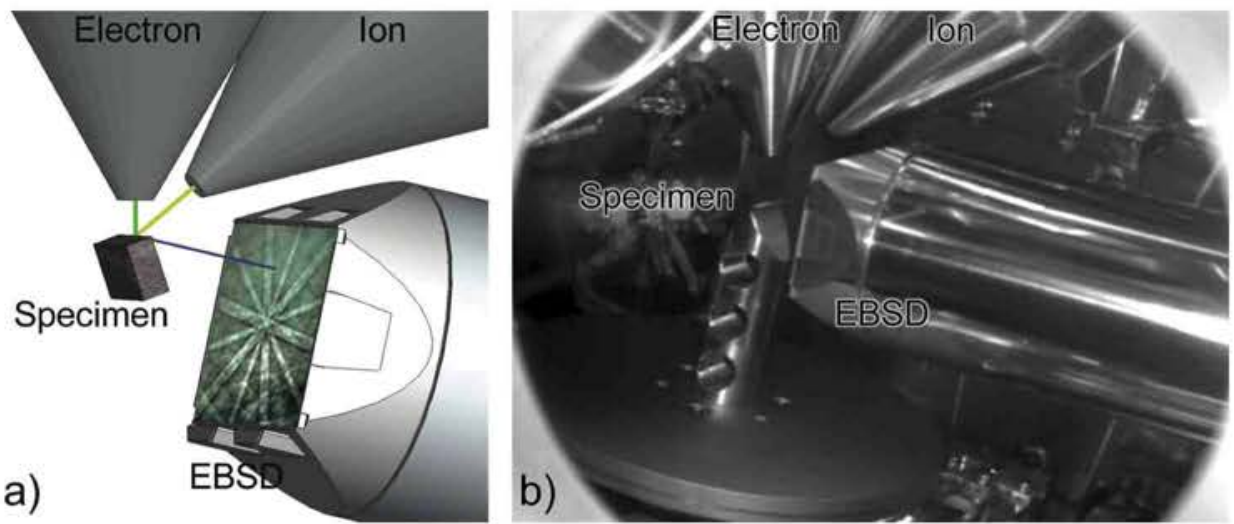

Fig 1a: Schematic showing the sample in the EBSD position. The electron beam is vertical, the ion beam is at $52^{\circ}$ to it, and the Nordlys EBSD detector is at $12^{\circ}$ below horizontal. Fig. 1b: Actual "live" view taken by the CCD camera mounted at the back of the chamber. The EBSD detector is inserted.

Electron Microscopy) tomography. The scale area in between these two techniques is commonly covered by EBSD. By extending the technique with a capability to access the third dimension, 3D crystallography is now also available at the micron / nano scale. This new modality is achieved by combining an electron beam for EBSD analysis with a focused ion beam for milling into the specimen to successively remove slices of the material of interest with subsequent
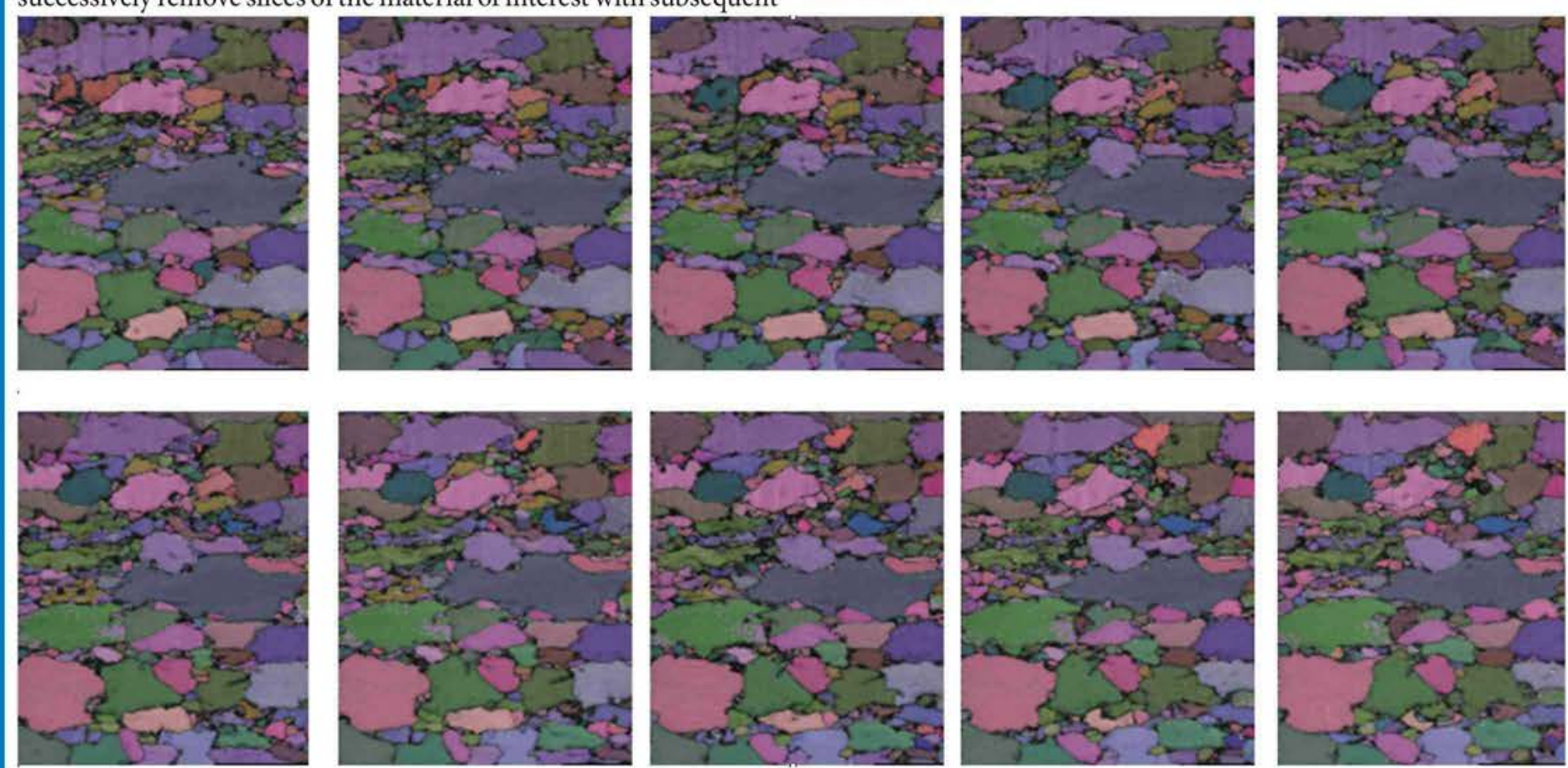

Figure 2: Successive EBSD maps on TRIP steel. Eight slices of a series of 62. Map size $15 \times 20 \mu \mathrm{m}$ slice distance $0.2 \mu \mathrm{m}$. Sample courtesy of Dr. Roumen Petrov (Ghent University, Belgium). 


\section{Universal Spectral Engine ${ }^{\mathrm{m}}$}

The leader in EDX and imaging systems innovation introduces the next revolution in microanalysis:

\section{RevolutionEDX ${ }^{\mathrm{Tm}}$}

The most significant advance in EDX since Spectrum Imaging.

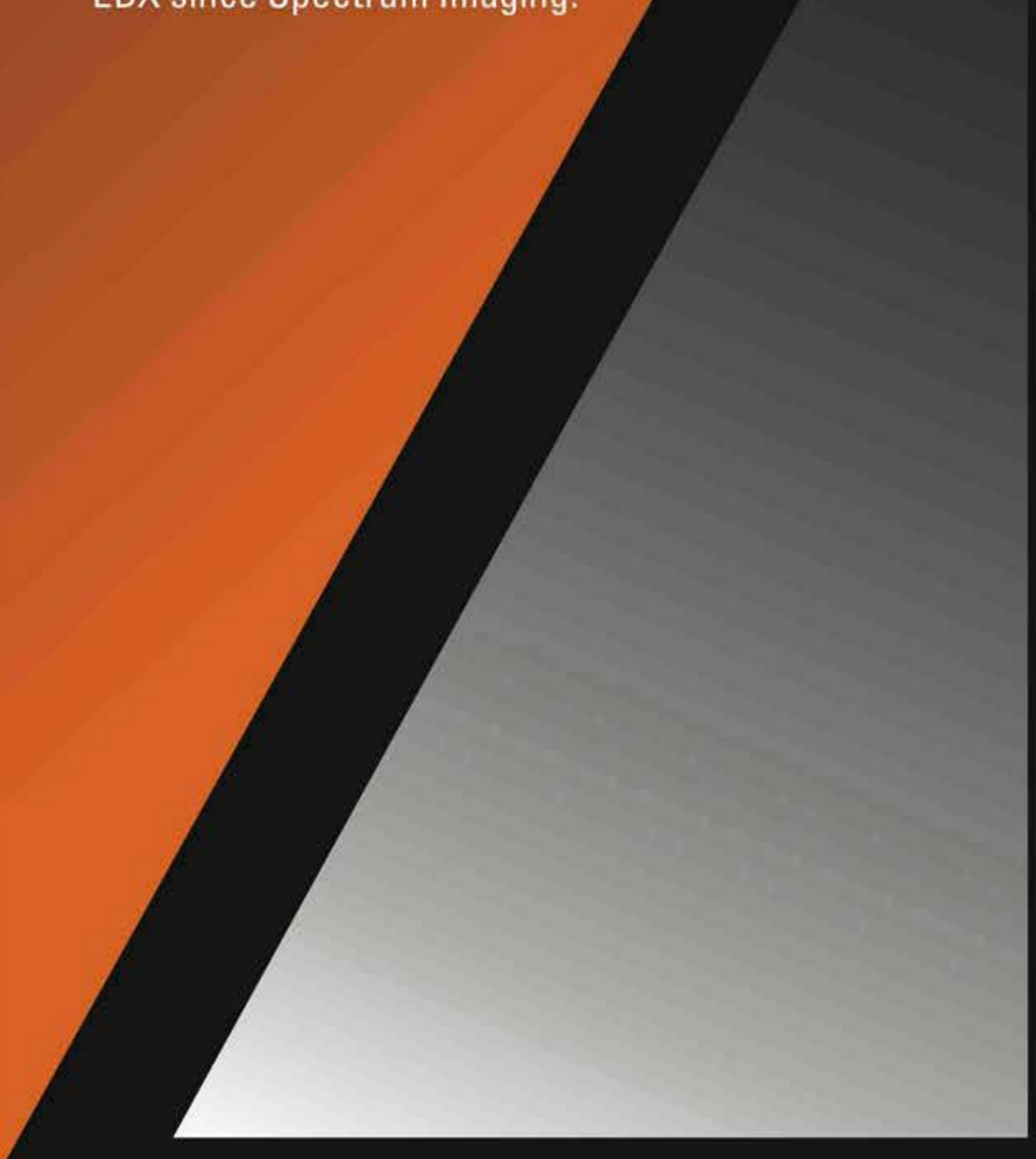

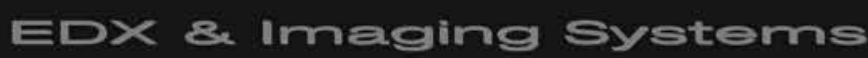

At the heart of RevolutionEDX'M is the Universal Spectral Engine: a new concept in Energy Dispersive X-Ray Microanalysis and Imaging.

See why we call this a revolution. Call us today to learn how integrating this remarkable new system into your workflow can save you time and add new capabilities and power to your electron microscope.

\section{Integrated by design}

All new electronics designed for high-speed transport of digital $x$-ray and electron event data

\section{The first Universal system}

A stand-alone network system that seamlessly interfaces to all $x$-ray detectors and SEMs/STEMs regardless of manufacturer

\section{Unique streaming technology}

Provides rapid 16-bit $\mathrm{x}$-ray and electron image collection for real-time analysis

\section{Join Us}

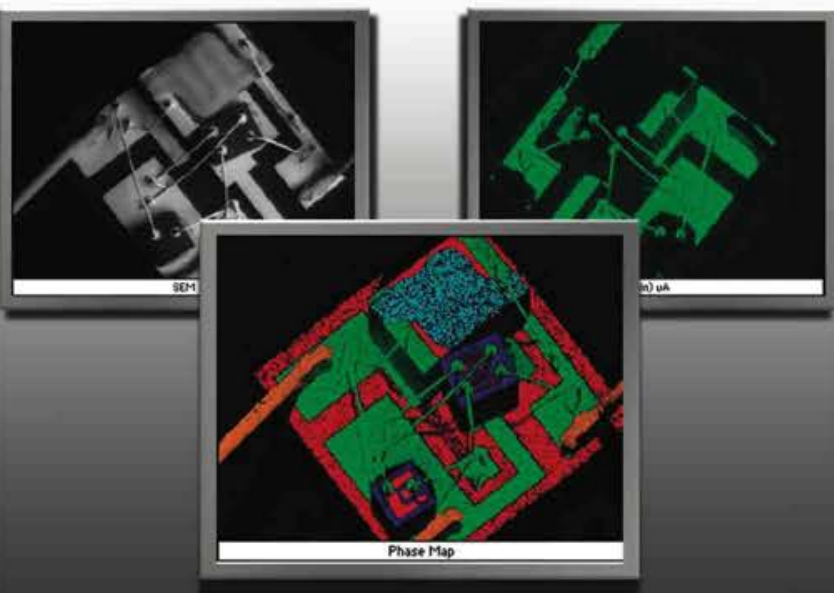

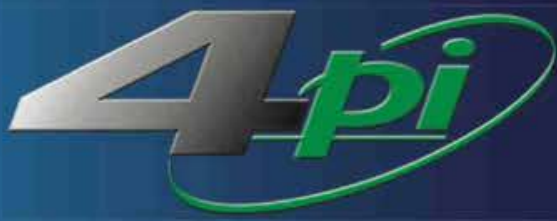

www. 4 pi.com

4pi Analysis, Inc.

(919) 489-1757• info@4pi.com 

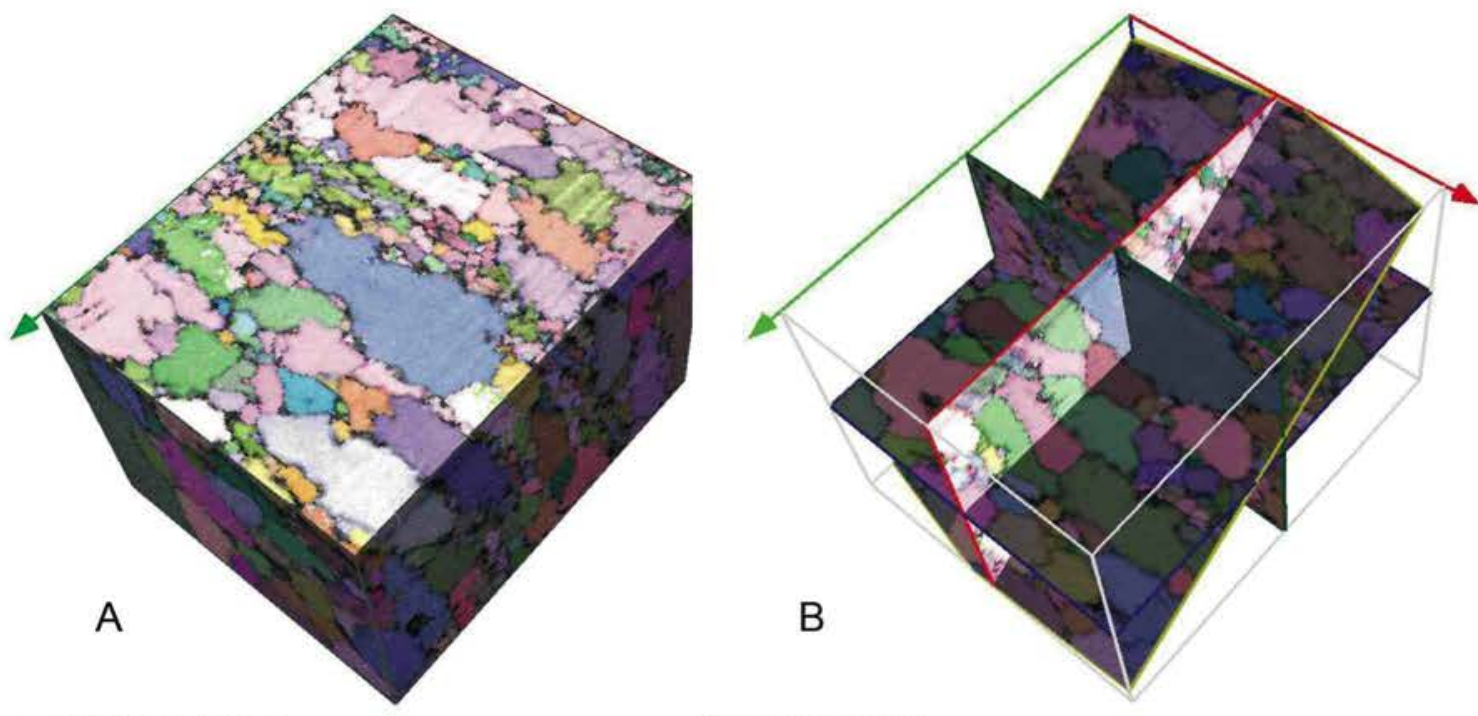

Figure 3: The assembled 3D map and the sectioned planes.

A few major characteristics that need understanding and discussion are:

\section{- Geometry}

For this DualBeam application, two distinct positions are identified:

1) "Mill location" where the specimen surface to be milled away is parallel to the ion beam, so that the slice can be removed by very low angle milling.

2) "EBSD location" where the specimen surface is at $20^{\circ}$ to the electron beam. This is the usual condition for EBSD analysis with good accuracy.

As the two stage positions are stored, it is possible to go from one position to the other in a matter of seconds. The re-positioning of the sample however, needs to be executed with a very high precision to allow successive maps to be aligned within the pixel resolution of the EBSD maps, and this can easily be as low as $10 \mathrm{~nm}$ (128 pixels on a 1 um field of view). Because the stage accuracy is not at this level, dedicated marker and pattern recognition technology has been applied to obtain this level of re-positioning accuracy.

In this way, there is never a direct line of sight between the ion column and the fluorescent screen, thus avoiding the possibility of screen damage which may lead to unacceptable charging, low yield, and black spots.

\section{- Shadowing}

As the milling proceeds into the sample, deeper areas become available for EBSD mapping. However, for proper indexing, the EBSD signal will have to reach the detector without any shadows (cut-off). Especially deep areas may suffer from shadowing of the edge of the cut-out for the series of slices being collected. Therefore, more material must be milled away than just for opening up the sample and for allowing access to the successive slices. This extra amount of material causes more than a doubling of the volume of interest. However, a gain in indexing accuracy is the real benefit of this approach.

\section{- Milling current}

The current of the ion beam is related to the spot size in a similar way that the electron beam current is related to a beam diameter. A typical removal yield is around $0.3-05 . \mu \mathrm{m}^{3} / \mathrm{nC}$ so a total volume of $20 \times 20 \times 20$ $\mu \mathrm{m}$ would require a total time of $16,000-27,000$ seconds, applying a beam current of $1 \mathrm{nA}$. A higher beam current would increase the milling speed, but it also may generate artefacts and hence make the surface less suited for direct, unambiguous EBSD analysis. A lower beam current (and beam energy) results in a slower milling process, but a better surface quality. The total process of milling can be speeded up by using a two-step process: coarse milling using a high current and fine milling using a low current / low energy. While the total milling time is driven by the size of the volume and the applied beam current(s), the number of slices is determined by the required resolution in the third dimension. Generally, an EBSD lateral resolution is matched with the grain sizes of interest. As in any three dimensional problem, depth resolution should be matched to lateral resolution and in this way the number of slices can easily be 128 or more. With an increasing number of slices, the total milling time is not changing, but the overall time will slightly increase due to additional positioning accuracy requirements, discussed below.

\section{- Electron beam current}

The electron beam current used for EBSD mapping should be as high as possible, in view of the desired lateral resolution. The beam current used for mapping has to be stable, and the same current must be used for the pattern recognition routine that defines the position of the map with respect to the applied markers. The applied mapping

(⿸丆口




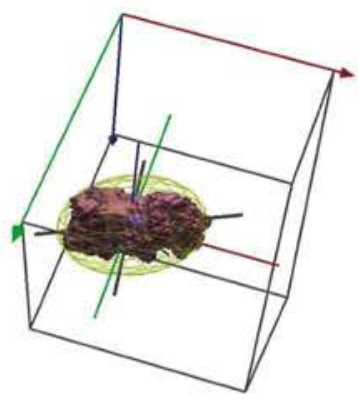

a)

b)

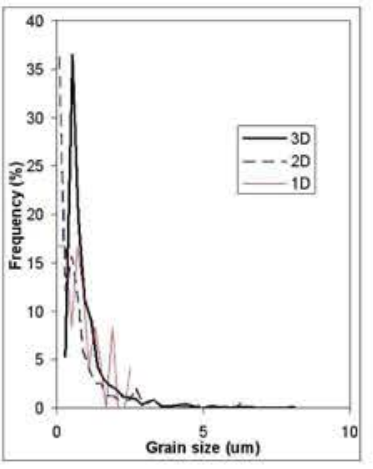

Figure 5a): a highlighted grain with the best ellipsoid fit and $b$ ): the grain size distribution calculated from $1 D, 2 D$ and $3 D$ measurement.

time is no different from the usual setting for 2D EBSD mapping and depends on the applied map size $x / y$, the camera constants and the required $\mathrm{S} / \mathrm{N}$ for the appropriate indexing. The system itself offers a beam current with a stability in the $0.5 \%$ range over the total length of the measurement.

\section{Automation}

The process of successive slicing, repositioning, and EBSD analysis can be executed manually i.e. by full on-site operator control. However, as the total process time can easily be 24 hours or more for a decent Z-resolution (many slices), this is not very practical. It will lead to errors, miscounts, and to low motivation for the operator. Therefore, it is very useful to automate the procedure, and this has been realized by the creation of software with a dedicated user interface: the ion beam control, the electron beam control, as well as the EBSD controls are fully embedded. The system parts of both the microscope and the EBSD system are transparent to the user, who does not have to bother with more complex scripting tasks. The system has a wizard type set-up with a simple checklist to guide the user through the necessary steps during the set-up phase and only shows the controls and graphics that are required at each step. The steps are detailed below:

1) The "set-up" phase (may take between 30 minutes and 1 hour): This phase provides the definition of the task at hand and includes direct feed-back of the validity of the chosen parameter setting. On-line check of the pattern recognition routines and indexing quality ensure that the definition of the job is correct and that the edge is cleaned prior to starting the automatic routine.

2) The "Run" phase (may take anywhere between 3 and 60 hours, depending on volume, map size, accuracy, material type and pattern validity):

The automation takes over full control of the DualBeam and EBSD system and the user's presence is no longer necessary. Images, EBSD orientations, and phase distributions are stored and up-to-date progress results are shown on the monitor.

Automation of the process is essential for the regular use of the technique because collection of any data set will be very time consuming. This is very similar to EBSD developments, where the availability of automatic indexing has really boosted the practical use of the technique to a wide variety of materials problems.

\section{Example of an automated run}

The example described here analyzed a trip steel sample and produced 62 maps during a week-end run of 55 hours (unattended). The sample had been electro-polished so the actual first ion beam clean-up cut could be kept to a minimum. Some of the resulting maps are shown in figure 2, for a slice distance of $200 \mathrm{~nm}$.
The successive $2 \mathrm{D}$ maps with $0.2 \mu \mathrm{m}$ step size can be assembled together using the Channel post-processing software to form a $3 \mathrm{D}$ map as shown in figure $3 \mathrm{a}$. Note that in a $3 \mathrm{D}$ map each data point is termed a 'Voxel' as opposed to 'Pixel' in 2D maps.

During the assembling step, the software can refine the alignment of layers by calculating offsets by comparing each layer to its neighbouring layers. Furthermore, the data can be filtered using classical methods ${ }^{[1]}$ utilizing the 26 nearest-neighbours for each voxel. ${ }^{[2]}$ The resulting $3 \mathrm{D}$ maps can be sectioned either in orthogonal planes of $X Y, Y Z, X Z$ or any arbitrary plane, as shown in figure $3 \mathrm{~b}$.

\section{Data processing}

The 3D map can be analysed to show the texture in the trip steel sample. In this dataset, it was found that there is a strong texture with $\{111\}$ parallel to the rolling direction, where the rolling direction is shown as $Y$ in the pole figure from the whole 3D data set, see figure $4 \mathrm{a}$. The grains with this texture component can be highlighted in a $3 \mathrm{D}$ map as shown in figure $4 \mathrm{~b}$, where these grains are shown in red.

A critical grain boundary of $10^{\circ}$ was chosen to do a grain reconstruction in $3 \mathrm{D}$, where for each grain a best fit ellipsoid is calculated: one of the grains is highlighted in figure $5 \mathrm{a}$. The orientation and dimensions of each grain can be statistically analysed. Figure $5 \mathrm{~b}$ compares the grain size distribution from the trip steel sample, calculated from spherical equivalent diameter (3D map), circular equivalent diameter (2D slices) and linear line intercept (1D lines) measurements. There is a lot of scatter in the grain size distributions calculated from the $1 \mathrm{D}$ and $2 \mathrm{D}$ data, whereas the $3 \mathrm{D}$ data shows a more statistically valid distribution. Table 1 compares the grain size measurements from each method, where the average grain sizes where found to be similar. However in this sample the grains are elongated and the 3D data is found to be a more reliable method for covering the grains to find the true maximum grain size.

\begin{tabular}{|c|c|c|}
\hline Method & $\begin{array}{c}\text { Average grain size } \\
(\mu \mathrm{m})\end{array}$ & Maximum grain size $(\mu \mathrm{m})$ \\
\hline 1D & 0.8 & 2.4 \\
\hline 2D & 0.7 & 6.2 \\
\hline 3D & 0.8 & 8.1 \\
\hline
\end{tabular}

Table 1: Grain size measurements using three methods of spherical equivalent diameter ( $3 D$ map), critical equivalent diameter ( $2 D$ slices) and linear line intercept (1D lines) measurements.

\section{Conclusions}

Three-dimensional crystallography of materials can be determined with a DualBeam (FIB / SEM) in combination with an EBSD detector and dedicated software. The automation of this process has been realized with a dedicated software solution including embedded control of the relevant functions. Data collection cycles with good $z$-resolution will require many slices and hence the total collection time is long. Automation permits convenient overnight and weekend analysis, when there is often reduced demand for the instrument. All EBSD maps are recorded and used for further data processing. The data processing of the $3 \mathrm{D}$ maps from the trip steel sample were able to show the true distribution and shape of the grains, whereas the 2D maps lack the full information for this analysis. The advantages of 3D maps over 2D maps for boundary analysis are discussed elsewhere ${ }^{[2-3]}$.

\section{References}

1- HKL Technology - Channel5 manual, Hobro, Denmark, 2004.

2- J.J.L. Mulders and A.P. Day, Materials Science Forum, Vol. 495-497, pp237-242, 2005.

3- Nave, M. D., Mulders, J. J. L., Gholinia, A., Twin characterisation using 2D and 3D EBSD, Chinese Journal of Stereology and Image Analysis, Vol. 10, No. 4, Dec. 2005, pp. 199-204. 